\title{
Evidência Psicométrica da Estrutura Fatorial do Sexismo Ambivalente em Profissionais de Psicologia do Brasil
}

\author{
Psychometric Evidence of Factorial Structure of \\ Ambivalent Sexism in Brazilian Psychologists \\ Icaro Bonamigo Gaspodini ${ }^{1}$ \\ Nilton Soares Formiga ${ }^{2}$ \\ Denise Falcke ${ }^{3}$ \\ ${ }^{1,3}$ Universidade do Vale do Rio dos Sinos São Leopoldo, Programa de Pós-Graduação \\ em Psicologia, RS, Brasil \\ ${ }^{2}$ Universidade Potiguar / Laureate International Universities Natal, Programa de Pós- \\ Graduação em Psicologia Organizacional e do, RN, Brasil
}

\begin{abstract}
Resumo. Uma perspectiva de avaliação do preconceito frente às mulheres pode ser obtida por meio do Inventário de Sexismo Ambivalente (ISA). O sexismo ambivalente é composto por duas formas de manifestação: hostil e benévola. O objetivo principal do presente estudo foi avaliar a estrutura fatorial do sexismo ambivalente em uma amostra de psicólogos/as no Brasil. Além disso, também se avaliou o sexismo ambivalente em relação à idade, ao tempo de atuação e ao quanto o profissional se identificava com uma lista de áreas de atuação. Responderam a um questionário online 497 profissionais, entre 22 e $69 \operatorname{anos}(M=34.52 ; D P=9.57)$, de todas as regiões do país. A análise fatorial confirmatória foi realizada por meio de diversos índices de qualidade de ajuste do modelo aos dados e as associações foram calculadas com correlações de Pearson. Os resultados mostraram indicadores psicométricos confiáveis para a estrutura bifatorial do sexismo ambivalente (hostil e benévolo) na amostra estudada. O sexismo esteve positivamente correlacionado com idade e não apresentou correlação com tempo de profissão. Quanto à identificação com a área de atuação, esteve negativamente correlacionado à Psicologia Social e Comunitária e positivamente correlacionado com Avaliação Psicológica, Neuropsicologia e Neurociência do Comportamento, Psicologia do Esporte, Psicologia do Trânsito, Psicologia Experimental e Psicologia Organizacional e do Trabalho.
\end{abstract}

Palavras-chave. Preconceito, discriminação, estereótipo de gênero, psicologia.

Abstract. A perspective to assess prejudice against women can be obtained through the Ambivalent Sexism Inventory (ASI). Ambivalent sexism consists of two forms of manifestation: hostile and benevolent. The main objective of this study was to evaluate the factorial structure of ambivalent sexism in a sample of Brazilian psychologists. In addition, ambivalent sexism was also evaluated in relation to age, time served and how much the professional identified with a list of psychology fields. A total of 497 psychologists, between the ages of 22 and 69 $(M=34.52 ; S D=9.57)$ from all regions of the country responded to an online survey. In addition to descriptive statistical analysis, the confirmatory factor analysis was carried out using several quality indices to adjust the model to the data, and the associations were calculated with Pearson's correlations. Results showed reliable psychometric scores for the bifactorial structure of ambivalent sexism (hostile and benevolent). Sexism was positively correlated with age and showed no correlations with time of occupation. As far as the identification with psychology fields, sexism was negatively correlated with Social and Community Psychology, and positively correlated with Psychological Evaluation, Neuropsychology and Behavioral Neurosciences, Sport Psychology, Traffic Psychology, Experimental Psychology and Organizational Psychology.

Keywords. Prejudice, discrimination, gender stereotypes, psychology.

${ }^{1}$ Icaro Bonamigo Gaspodini. Universidade do Vale do Rio dos Sinos São Leopoldo. Programa de Pós-Graduação em Psicologia, Av. Unisinos, 950, Bairro Cristo Rei, RS, Brasil. E-mail: icaroicaro@gmail.com

${ }^{2}$ Nilton Soares Formiga. Universidade Potiguar / Laureate International Universities Natal. Programa de Pós-Graduação em Psicologia Organizacional e do , RN, Brasil. E-mail: nsformiga@yahoo.com

${ }^{3}$ Denise Falcke. Universidade do Vale do Rio dos Sinos São Leopoldo. Programa de Pós-Graduação em Psicologia, RS, Brasil. E-mail: dfalcke@unisinos.br

\section{(c) $($ †) $\ominus$}

Esta obra está bajo una licencia de Creative Commons Reconocimiento-NoComercial-SinObraDerivada 4.0 Internacional. 


\section{Introdução}

Atitudes de segregação em relação a minorias sociais têm se revelado através de condutas menos diretas, o que aumenta a percepção de que o preconceito tem acabado (Formiga, Omar, \& Vaamonde, 2009). O que acontece, na verdade, é que nas últimas décadas, normas sociais passaram a inibir expressões discriminatórias contra minorias sociais, tornando essas expressões mais sutis (Pereira, Monteiro, \& Camino, 2009). Obviamente, segundo Myers (2014), julgar ou atribuir diferenças é algo implícito no sujeito, podendo ser difícil de inibir, devido ao seu caráter cognitivo, mas é possível gerar controle de comportamentos discriminatórios, condição para a qual as normas contra a discriminação têm contribuído.

Nesse sentido, uma breve distinção entre preconceito e discriminação faz-se necessária. De forma geral, o termo "discriminação" se refere a um comportamento, uma ação que reforça os estereótipos e rótulos contidos no preconceito (Pescosolido \& Martin, 2015). O preconceito é uma dimensão de crenças e atitudes negativas frente a um indivíduo pertencente a um grupo cujas características são consideradas negativas em determinada sociedade. O indivíduo discrimina (age) com base no preconceito; portanto, ainda que a discriminação venha sendo atenuada por normas sociais, o mesmo não acontece com o preconceito, que assumiu formas cada vez mais sutis, camufladas, disfarçadas e difíceis de serem observadas (Formiga, 2007; Formiga \& Silva Neta, 2009; Pettigrew \& Meertens, 1995; 2001).

Uma das formas de preconceito ainda muito presentes atualmente é o sexismo, um conjunto de crenças e atitudes sobre mulheres, que envolvem atributos estereotipados de cuidado, passividade, delicadeza e subserviência (Formiga \& Camino, 2001). Para auxiliar na compreensão da dinâmica social desse fenômeno, Glick e Fiske (1996) propõem o conceito de "sexismo ambivalente". O caráter ambivalente do sexismo se deve ao fato de suas manifestações "não serem tão diretas e claras como as posturas mais tradicionais de discriminação, geralmente baseadas na assumida inferioridade ou diferença das mulheres como um grupo" (Formiga, Gouveia, \& Santos, 2002, p. 105). Essa condição sugere duas dimensões para que o sexismo seja compreendido: o sexismo hostil e o sexismo benévolo. O sexismo hostil, uma expressão flagrante de preconceito, se refere à atitude de antipatia e intolerância frente às mulheres, com base na crença de sua inferioridade frente aos homens. Já sexismo benévolo seria o tratamento diferenciado para mulheres, de certa forma disfarçado de atitude positiva, mas que a posiciona como um ser frágil, que precisa de atenção e provisão masculina (Glick \& Fiske, 1996; 2001; Pettigrew \& Meertens, 1995).

Para avaliar esse constructo, Glick e Fiske (1996) desenvolveram o Inventário de Sexismo Ambivalente (ISA). De acordo com os autores, cada uma das dimensões do sexismo (hostil e benévolo) são formadas por três subdimensões: o paternalismo, a diferenciação de gênero e a heterossexualidade (Glick \& Fiske, 1996; Mladinic, Saiz, Díaz, Ortega, \& Oyarce, 1998). O paternalismo se apresenta de duas formas, denominadas paternalismo dominante e paternalismo protetor. $\mathrm{O}$ primeiro se refere ao tratamento das mulheres como incapazes, como se necessitassem aporte de uma suposta superioridade masculina; o paternalismo dominante está relacionado com o sexismo hostil. O segundo envolve uma noção de debilidade frente às mulheres, como se necessitassem da proteção dos homens; $\mathrm{O}$ 
paternalismo protetor está relacionado ao sexismo benévolo. Na diferenciação de gênero, o sexismo hostil se manifesta na competição, quando se considera que homens possuam as características necessárias à direção e tomada de decisão. Por outro lado, o sexismo benévolo confere atributos positivos à mulher, mas cuja legitimidade se condiciona a ser complementar aos atributos masculinos. Por fim, a heterossexualidade apresenta, de um lado a dinâmica da dominação sexual (sexismo hostil) e de outro a atribuição de compaixão e necessidade de ajuda para uma mulher que não tenha um companheiro (sexismo benévolo).

O ISA foi adaptado ao contexto brasileiro, em uma amostra de jovens universitários/as do estado da Paraíba, por Formiga et al. (2002). Nesse estudo, foram confiáveis os índices de consistência interna e relação itens-fator, o que também foi confirmado em pesquisas subsequentes por Ferreira (2004) e Formiga (2005, 2011, 2015). No estudo mais recente, utilizou-se uma amostra de 795 pessoas de 18 a 47 anos, da cidade de João Pessoa, Paraíba (Formiga, 2015). Foram observados indicadores psicométricos que corroboram o axioma teórico apontado por Glick e Fiske (1996), autores do ISA.

Sendo o sexismo ambivalente, tanto em sua medida, quanto em sua teoria, uma perspectiva para avaliar o preconceito em relação às mulheres em distintos contextos, observou-se que o instrumento que o mensura (ISA) tem sido aplicado, especificamente, em amostras de universitários e na população geral. Além disso, sabe-se sobre a importância de avaliar a variação e o nível de preconceito contra mulheres em diferentes categorias grupais, pois elas contribuiriam tanto para regulação e institucionalização de políticas antissexistas, quanto para o reconhecimento e manutenção de uma prática profissional com base nos direitos humanos. Um desses importantes grupos é representado pelas psicólogas e psicólogos do Brasil. No site "A Psicologia brasileira apresentada em números", mantido pelo Conselho Federal de Psicologia, consta um número de 339596 profissionais registrados, atualizado no dia 22/04/2019. Em todos os estados brasileiros, as mulheres são maioria na profissão (Conselho Federal de Psicologia, 2019).

A avaliação do sexismo ambivalente nessa amostra também se justifica pelo fato de que crenças e atitudes pessoais do/a psicólogo/a estão em estreita relação com as práticas que realizam em seus diversos contextos de atuação. No Brasil, sua conduta se baseia no Código de Ética Profissional do Psicólogo, do Conselho Federal de Psicologia (2005), o qual foi inspirado nas diretrizes da Declaração Universal dos Direitos Humanos (Giovannetti, 2009), bem como nos princípios científicos e epistemológicos da profissão (Camino, 1998). Uma das mais importantes diretrizes se refere ao fato de que são vedadas quaisquer formas de preconceito e discriminação no exercício da profissão, estando os/ as profissionais obrigados a eliminar "quaisquer formas de negligência, discriminação, exploração, violência, crueldade e opressão" (Conselho Federal de Psicologia, 2005, p. 7). Poucas pesquisas avaliam formas de manifestação de preconceito em amostras de psicólogos/as no Brasil. Recentemente, no entanto, encontrou-se a presença de preconceito extremo contra diversidade sexual e de gênero nessa população (Gaspodini \& Falcke, 2018a). O estudo mostrou que crenças de base psicológica sobre a natureza da homossexualidade, bissexualidade e transexualidade predisseram a maior ocorrência de preconceito contra diversidade, em detrimento de crenças de base religiosa, ético-moral 
ou biológica. Entre essas crenças estão conhecimentos tradicionalmente encontrados em algumas teorias psicológicas, que explicariam "causas" da diversidade sexual com base na perversão do comportamento sexual, abuso sexual sofrido na infância ou má resolução com figuras parentais. Estudo subsequente indicou a presença de estereótipos ligados à feminilidade e expressões sutis de sexismo em psicólogas no Rio Grande do Sul (Gaspodini \& Falcke, 2018b).

Frente a isso, o objetivo principal do presente estudo foi avaliar a estrutura fatorial do sexismo ambivalente em uma amostra de psicólogos/as no Brasil. Além disso, também se avaliou o sexismo ambivalente em relação à idade, ao tempo de atuação e ao quanto o profissional se identificava com uma lista de áreas de atuação.

\section{Método}

\section{Participantes}

Participaram do estudo, 497 psicólogos/as brasileiros/as, com idades entre 22 e 69 anos $(M=34.52 ; D P=9.57)$, credenciados em 22 dos 23 Conselhos Regionais de Psicologia do país. Com o objetivo de garantir a qualidade da amostra, utilizou-se o pacote estatístico G*Power 3.1 para calcular o poder estatístico, tendo como base o $n$ necessário para a pesquisa e o tipo de cálculo a ser realizado (Faul, Erdfelder, Buchner, \& Lang, 2007). Com isso, em relação à coleta de dados do estudo, considerou-se uma probabilidade de $95 \%$ $(p<.05)$, magnitude do efeito amostral $(t) \geq .30$ e um padrão de poder hipotético $(\pi) \geq$ 0.80 . Gerado o cálculo amostral, com base nesses critérios, a amostra coletada revelou-se suficiente e significativa para o presente estudo, tendo como indicadores: $t \geq 1.98 ; \pi \geq$ $1.00 ; p<.05)$. Na tabela 1, foram apresentadas as principais características da amostra.

\section{Instrumentos}

Inventário de Sexismo Ambivalente (IS A). Desenvolvido por Glick e Fiske (1996), o instrumento é composto por 22 itens nos quais o indivíduo deve assinalar apenas uma opção numa escala de cinco pontos: (1) discordo totalmente, (2) discordo, (3) nem discordo, nem concordo (4) concordo, e (5) concordo totalmente. Esse inventário tem como objetivo avaliar ideias sexistas, quer de um ponto de vista benévolo, quer de um ponto de vista hostil.

Esses 22 itens estão distribuídos em dois fatores. O fator 1 (itens: 2, 4, 5, 7, 10, 11, 14, 15, 16, 18 e 21) reflete o sexismo hostil (p.ex., "As mulheres feministas estão fazendo exigências completamente sem sentido aos homens"; "A maioria das mulheres não apreciam completamente tudo o que os homens fazem por elas"), uma vez que demonstra um preconceito mais evidente face ao gênero feminino. $O$ fator 2 (itens: $1,3,6,8,9$, 12, 13, 17, 19, 20 e 22) diz respeito ao sexismo benévolo (p.ex., "As mulheres devem ser queridas e protegidas pelos homens"; "Muitas mulheres se caracterizam por uma pureza que poucos homens possuem"), na medida em que demonstra uma forma sutil de diferenciação das mulheres.

No Brasil, o ISA tem apresentado indicadores psicométricos muito semelhantes aos observados em outros estudos de distintos países, como Estados Unidos, Chile, México, Coreia e Alemanha. Especificamente, Formiga et al. (2002), Formiga (2005) e Formiga, 
Tabela 1

Características da Amostra

\begin{tabular}{|c|c|c|}
\hline Variável & \% válida & $n$ \\
\hline \multicolumn{3}{|l|}{ Gênero } \\
\hline Feminino & 79.7 & 396 \\
\hline Masculino & 19.5 & 97 \\
\hline Outros & 0.8 & 4 \\
\hline \multicolumn{3}{|l|}{ Local de moradia } \\
\hline Capital & 41.9 & 208 \\
\hline Interior & 41.1 & 204 \\
\hline Região metropolitana & 16.9 & 84 \\
\hline \multicolumn{3}{|l|}{ Região de atuação } \\
\hline Sul & 67.4 & 317 \\
\hline Sudeste & 21.7 & 102 \\
\hline Nordeste & 6.8 & 32 \\
\hline Centro-Oeste & 2.8 & 13 \\
\hline Norte & 1.3 & 6 \\
\hline \multicolumn{3}{|l|}{ Tempo de atuação } \\
\hline Entre alguns meses até 10 anos & 70.6 & 346 \\
\hline Mais de dez anos & 29.4 & 144 \\
\hline \multicolumn{3}{|l|}{ Formação completa } \\
\hline Especialização & 40.2 & 198 \\
\hline Mestrado Acadêmico & 25 & 123 \\
\hline Graduação & 23.4 & 115 \\
\hline \multicolumn{3}{|l|}{ Formação em andamento } \\
\hline Nenhuma & 43.8 & 208 \\
\hline Especialização & 23.6 & 112 \\
\hline Mestrado Acadêmico & 14.1 & 67 \\
\hline Doutorado e Pós-Doutorado & 13.1 & 62 \\
\hline
\end{tabular}


Guedes, Silva e Oliveira (2012) revelaram resultados que mantêm uma constante psicométrica confiável, na qual seus indicadores estatísticos têm se organizado de forma simétrica em sua temporalidade e em diferentes contextos sociais, políticos e etários.

Dados sociodemográficos e da profissão. Além da idade e do gênero dos/as participantes, avaliouse o tempo de atuação como psicólogo/a e também a identificação do/a profissional com uma lista de áreas de atuação. Nesse caso, o/a participante deveria indicar em uma escala de cinco pontos $(1=$ discordo totalmente $/ 5=$ concordo totalmente $)$ o quanto se identificava com cada uma das seguintes áreas: Análise Institucional, Neurociência do Comportamento, Psicologia Clínica, Psicologia da Saúde, Psicologia das Emergências e Desastres, Psicologia do Desenvolvimento Humano, Psicologia do Esporte, Psicologia do Trânsito, Psicologia Escolar e Educacional, Psicologia Experimental, Psicologia Hospitalar, Psicologia Jurídica ou Forense, Psicologia na Área das Deficiências, Psicologia Organizacional e do Trabalho, Psicologia Política, Psicologia Social e Comunitária, Sexualidade Humana, Uso Abusivo de Substâncias. Quanto maior a pontuação na escala, maior a identificação do/a psicólogo/a com cada uma das áreas de atuação.

\section{Procedimentos}

Foram enviados cerca de 2.5 mil convites por e-mail e compartilhados em redes sociais para universidades, instituições de formação e empresas de Psicologia. O Conselho Regional de Psicologia do Rio Grande do Sul divulgou o convite em sua página oficial e o do Espírito Santo convidou todos os cadastrados por mala direta.

Um questionário online contendo os instrumentos previamente descritos foi construído na plataforma Formulários Google e disponibilizado por 46 dias, entre 15/03/16 e 29/04/16. Para garantir a leitura e compreensão do Termo de Consentimento Livre e Esclarecido (TCLE), foi criada uma subseção na qual se encontrava a única caixa de diálogo marcada como obrigatória. Para respeitar o direito de desistência de participantes, além da opção "Sim, eu li e compreendi o TCLE e aceito participar desta pesquisa", foi incluído "Não aceito participar desta pesquisa" - levando ao fim do questionário. Em caso de dúvida, participantes foram instruídos a contatar o pesquisador responsável. Foram informados os benefícios da participação, como a colaboração com o progresso científico e a oportunidade de expressar opiniões, bem como os riscos de eventual desconforto com algum tema. No último caso, participantes poderiam, além de abandonar a pesquisa sem prejuízo, entrar em contato com o pesquisador responsável para comunicar a razão do desconforto.

Foram atendidas as diretrizes e normas para pesquisas envolvendo seres humanos do Conselho Nacional de Saúde (CNS), de acordo com a Resolução 466, de 12 de dezembro de 2012. Este estudo foi aprovado pelo Comitê de Ética em Pesquisa da Universidade do Vale do Rio dos Sinos e pode ser conferido pelo CAAE 51286115.6.0000.5344.

\section{Análise dos dados}

Efetuou-se uma Análise Fatorial Confirmatória (AFC), gerando o Modelo de Equação Estrutural (MEE) a partir do AMOS GRAFICS (versão 21.0) para comprovar, com maior robustez, a estrutura do ISA em uma amostra de profissionais na área da psicologia. Para 
realização da análise fatorial confirmatória, pretendeu-se verificar a adequação do modelo quanto a três propostas fatoriais: a unifatorialidade, a bidimensionalidade ortogonal e bidimensionalidade oblíqua. A matriz de covariâncias foi considerada como entrada, tendo sido adotado o estimador Maximum Likelihood (ML), o que permite uma análise estatística mais criteriosa e rigorosa em comparação à análise fatorial dos Principais Componentes (PC), utilizada nos estudos supracitados. Isso permite verificar diretamente uma estrutura fatorial teórica, como a proposta observada pelos autores que adaptaram o mesmo inventário em outros países.

A presente análise faz uso de alguns índices que permitem avaliar a qualidade de ajuste do modelo proposto (Bilich, Silva, \& Ramos, 2006; Byrne, 1989; Hair, Black, Babin, Anderson, \& Tatham, 2009; Joreskög \& Sörbom, 1989; Vijver \& Leung, 1997), conforme a seguir:

- Qui-Quadrado $\left(\chi^{2}\right)$. Testa a probabilidade do modelo teórico se ajustar aos dados. O ajustamento será pior conforme o valor seja maior. Pouco empregado na literatura, é mais comum considerar sua razão em relação ao grau de liberdade $\left(\chi^{2} / g \emptyset\right.$. Nesse caso, valores até 3 indicam um ajustamento adequado.

- Rair Quadrada Média Residual Saturado (RMRst). Indica o quanto um modelo teórico se ajusta aos dados, na medida em que a diferença entre os dois se aproxima de zero.

- Goodness-of-Fit Index (GFI) e Adjusted Goodness-of-Fit Index (AGFI). Considerados análogos ao $\mathrm{R}^{2} \mathrm{em}$ regressão múltipla, indicam a proporção de variância-covariância nos dados explicada pelo modelo, que variam de 0 a 1 , com valores na casa dos $.80 \mathrm{e}$ .90 , ou superior, indicando um ajustamento satisfatório.

- Comparative Fit Index (CFI). Compara o modelo estimado e o modelo nulo de forma geral, considerando valores mais próximos de 1 como indicadores de ajustamento satisfatório.

- Tucker-Lewis Index (TLI). Fornece uma medida de parcimônia entre os índices do modelo proposto e do modelo nulo. Varia de 0 a 1, com índice aceitável acima de .90 .

- Root-Mean-Square Error of Approximation (RMSEA). Com intervalo de confiança de 90\%, é considerado um indicador de "maldade" de ajuste, isto é, valores altos indicam um modelo não ajustado. Assume-se como ideal que o RMSEA se situe entre $.05 \mathrm{e}$ .08 , aceitando-se valores de até .10 .

- Expected Cross-Validation Index (ECVI) e Consistent Akaike Information Criterion (CAIC). Indicadores geralmente empregados para avaliar a adequação de um modelo determinado em relação a outro. O melhor ajuste é expressado por valores baixos do ECVI e CAIC.

- Akaike's Information Criteria (AIC). Avalia o modelo utilizando a parcimônia, levando em conta o número de parâmetros estimados. É utilizado quando compara-se dois ou mais modelos. O modelo que apresenta melhor ajuste é o que possuiu menor AIC. 
- Browne-Cudeck Criterion (BCC). Funciona da mesma maneira que o AIC e o CAIC, com a diferença de que impõe grandes penalidades para a complexidade do modelo.

- Bayes Information Criterion (BIC). Mostra-se de forma mais consistente, uma vez que cada ajuste realizado nos componentes explicativos é gerado com base no ajuste adequado dos dados, penalizando severamente modelos com muitos parâmetros.

Além dos supracitados, também se calculou a confiabilidade composta (CC) e a variância média extraída (VME). No primeiro indicador, exige-se que o nível do escore seja acima de .70, enquanto que no segundo, é preciso um nível acima de .50 (Campana, Tavares, Silva, \& Diogo, 2009; Hair et al., 2009; Valentini \& Damásio, 2016).

Por fim, foram calculadas as correlações entre o sexismo ambivalente e a idade, tempo de atuação e identificação com a área de atuação profissional por meio de um teste de correlação de Pearson.

\section{Resultados}

A fim de atender aos objetivos do presente estudo, tomou-se como base de orientação teórico-empírica o modelo original de Glick e Fiske (1996), especificamente, o modelo bifatorial, o qual avalia as dimensões do sexismo hostil e benévolo interdependentes. Com isso, geraram-se os modelos unifatorial (considerando todos os itens somados organizando uma dimensão de sexismo) e bifatorial ortogonal (considerando a existência de dois fatores - um hostil e outro benévolo - não associados). Ao se tentar provar o modelo bifatorial oblíquo, que indicaria a existência dos dois tipos de sexismo inter-relacionados, os resultados revelaram que o modelo hipotetizado mostrou indicadores psicométricos melhores do que aqueles observados nos outros modelos fatoriais (tabela 2).

Além dos indicadores psicométricos observados, na tabela 3, constam outros parâmetros mais parcimoniosos (AIC, BIC e BCC), os quais garantiram o modelo hipotetizado. Isso ocorre justamente por serem resultados que acompanham o CAIC e ECVI, os quais são utilizados para avaliar a adequação de um modelo determinado em relação a outros modelos para comparação fatorial - neste caso, a proposta do modelo bifatorial oblíquo ajustado em relação aos modelos bifatorial ortogonal, oblíquo não ajustado e unifatorial. Com isso, observou-se que o modelo bifatorial oblíquo ajustado (M4) apresentou os melhores resultados em comparação às estruturas fatoriais dos outros modelos, o que corrobora a organização previamente encontrada em estudos sobre o sexismo no Brasil (cf. Formiga et al., 2002; Ferreira, 2004; Formiga, 2005; 2011).

$\mathrm{Na}$ tabela 4 , pode-se observar que todas as saturações (Lambdas, $\lambda$ ) estiveram dentro do intervalo esperado $|0-1|$, denotando não haver problemas com a estimação proposta, com todas estatisticamente diferentes de zero $(t>1.96, p<.05)$, garantindo a qualidade da validade estrutural. Também foram observadas associações Phi $(\Phi)$ positivas entre os fatores do sexismo hostil e benévolo $(\Phi=.75)$.

Quanto à validade do constructo do sexismo ambivalente, realizou-se os cálculos da CC e da VME. Observou-se que, para as dimensões do sexismo hostil, o CC e o $\mathrm{VME}$ estiveram acima do exigido na literatura $(\mathrm{CC}=.91$; $\mathrm{VME}=.58)$, ocorrendo 
Tabela 2

Indicadores Psicométricos da Estrutura Fatorial do Inventário de Sexismo Ambivalente

\begin{tabular}{|c|c|c|c|c|c|c|c|c|c|}
\hline \multirow[b]{2}{*}{ Modelos } & \multicolumn{4}{|c|}{ Medidas de ajuste absoluto } & \multicolumn{3}{|c|}{$\begin{array}{l}\text { Medidas de ajuste } \\
\text { incremental }\end{array}$} & \multicolumn{2}{|c|}{$\begin{array}{c}\text { Medidas de ajuste } \\
\text { parcimonioso }\end{array}$} \\
\hline & $x^{2} / g l$ & $\mathrm{RMR}_{\text {st }}$ & GFI & AGFI & CFI & TLI & $\begin{array}{c}\text { RMSEA } \\
\text { (Intervalo) }\end{array}$ & CAIC & $\begin{array}{c}\text { ECVI } \\
\text { (Intervalo) }\end{array}$ \\
\hline Modelo $^{1}$ & 6.92 & .08 & .77 & .69 & .77 & .75 & $\begin{array}{c}.11 \\
(0.10-0.12)\end{array}$ & 1764.17 & $\begin{array}{c}3.09 \\
(2.86-3.34)\end{array}$ \\
\hline Modelo $^{2}$ & 6.25 & .27 & .80 & .75 & .80 & .77 & $\begin{array}{c}.10 \\
(0.10-0.11)\end{array}$ & 1624.80 & $\begin{array}{c}2.81 \\
(2.59-3.05)\end{array}$ \\
\hline Modelo $^{3}$ & 5.91 & .13 & .80 & .75 & .81 & .79 & $\begin{array}{c}.10 \\
(0.09-0.11)\end{array}$ & 1552.77 & $\begin{array}{c}2.67 \\
(2.45-2.90)\end{array}$ \\
\hline Modelo $^{4}$ & 1.76 & .03 & .95 & .92 & .98 & .97 & $\begin{array}{c}.03 \\
(0.03-0.05)\end{array}$ & 914.73 & $\begin{array}{c}.94 \\
(0.85-1.05)\end{array}$ \\
\hline
\end{tabular}

Nota. ${ }^{1}$ Modelo unifatorial; ${ }^{2}$ Modelo bifatorial ortogonal; ${ }^{3}$ Modelo bifatorial oblíquo não ajustado; ${ }^{4}$ Modelo bifatorial oblíquo ajustado.

Tabela 3

Indicadores Psicométricos de Parcimônia para a Comparação da Estrutura Fatorial-Conceitual do Inventário de Sexismo Ambivalente

\begin{tabular}{cccc}
\hline & \multicolumn{3}{c}{ Indicador de parcimônia } \\
Modelos & AIC & BIC & BCC \\
\hline Modelo $^{1}$ & 1764.17 & 1720.17 & 1539.27 \\
Modelo $^{2}$ & 1395.62 & 1580.80 & 1399.90 \\
Modelo $^{3}$ & 1323.59 & 1508.77 & 1327.87 \\
Modelo $^{4}$ & 466.79 & 828.73 & 475.15
\end{tabular}

Nota. ${ }^{1}$ Modelo unifatorial; ${ }^{2}$ Modelo bifatorial ortogonal; ${ }^{3}$ Modelo bifatorial oblíquo não ajustado; ${ }^{4}$ Modelo bifatorial oblíquo ajustado. 
Tabela 4

Estrutura Fatorial do Inventário de Sexismo Ambivalente

\begin{tabular}{|c|c|c|c|c|c|}
\hline$\xi$ & $x$ & $\lambda$ & $\varepsilon$ & $\mathrm{CC}$ & VME \\
\hline \multirow[t]{11}{*}{ Sexismo hostil (SH) } & SH1 & .60 & .35 & & \\
\hline & SH2 & .61 & .26 & & \\
\hline & SH3 & .69 & .45 & & \\
\hline & $\mathrm{SH} 4$ & .70 & .49 & & \\
\hline & SH5 & .72 & .52 & & \\
\hline & SH6 & .75 & .54 & .91 & .58 \\
\hline & $\mathrm{SH7}$ & .68 & .46 & & \\
\hline & SH8 & .72 & .52 & & \\
\hline & SH9 & .77 & .47 & & \\
\hline & SH10 & .61 & .37 & & \\
\hline & SH11 & .76 & .43 & & \\
\hline \multirow[t]{11}{*}{ Sexismo benévolo (SB) } & SB1 & .69 & .48 & & \\
\hline & SB2 & .79 & .15 & & \\
\hline & SB3 & .68 & .46 & & \\
\hline & SB4 & .82 & .37 & & \\
\hline & SB5 & .68 & .33 & & \\
\hline & SB6 & .77 & .59 & .92 & .53 \\
\hline & SB7 & .77 & .56 & & \\
\hline & SB8 & .68 & .46 & & \\
\hline & SB9 & .67 & .33 & & \\
\hline & SB10 & .71 & .51 & & \\
\hline & SB11 & .70 & .33 & & \\
\hline
\end{tabular}

Nota. $\xi=$ Constructo psicológico; $\lambda=$ Escores fatoriais da estrutura; $\chi=$ Variáveis (itens); $\varepsilon=$ Erros de medida da estrutura; $\mathrm{CC}=$ Confiabilidade composta; VME = Variância média extraída.

o mesmo para o sexismo benévolo $(\mathrm{CC}=.92$; VME $=.52$ ). Tais resultados evidenciam tanto a confiabilidade quanto a validade convergente do constructo avaliado, justificando a adequabilidade da estrutura fatorial da medida do sexismo ambivalente em psicólogos.

Tais resultados, foram confirmados ao se observar as estimativas de predição, a partir da análise de regressão revelada para o modelo proposto. $\mathrm{Na}$ tabela 5 , identifica-se que as variáveis significativas e a razão critério estiveram dentro do que é estatisticamente exigido e estatisticamente diferentes de zero $(t>1.96, p<.05)$. Esses resultados 
Tabela 5

Indicadores das Estimativas Preditivas entre Itens-Fatores do Inventário de Sexismo Ambivalente

\begin{tabular}{|c|c|c|c|c|c|c|}
\hline Itens & Fatores & Estimativa & $D P$ & $\begin{array}{c}\text { Razão } \\
\text { Critério }\end{array}$ & $p<$ & VIF \\
\hline ISASH1 & $\mathrm{SH}$ & 1.00 & --- & --- & --- & 2.10 \\
\hline ISASH2 & $\mathrm{SH}$ & 0.82 & .08 & 10.36 & .001 & 1.20 \\
\hline ISASH3 & $\mathrm{SH}$ & 1.00 & .08 & 12.46 & .001 & 2.41 \\
\hline ISASH4 & $\mathrm{SH}$ & 1.03 & .08 & 12.76 & .001 & 1.89 \\
\hline ISASH5 & $\mathrm{SH}$ & 0.95 & .07 & 13.04 & .001 & 1.58 \\
\hline ISASH6 & $\mathrm{SH}$ & 1.12 & .08 & 13.35 & .001 & 2.95 \\
\hline ISASH7 & $\mathrm{SH}$ & 0.82 & .07 & 12.29 & .001 & 3.73 \\
\hline ISASH8 & $\mathrm{SH}$ & 1.04 & .08 & 12.98 & .001 & 1.73 \\
\hline ISASH9 & $\mathrm{SH}$ & 1.05 & .08 & 13.38 & .001 & 1.89 \\
\hline ISASH10 & $\mathrm{SH}$ & 1.13 & .10 & 11.44 & .001 & 1.92 \\
\hline ISASH11 & $\mathrm{SH}$ & 1.02 & .08 & 12.29 & .001 & 1.79 \\
\hline ISASB1 & SB & 1.00 & --- & --- & --- & 1.68 \\
\hline ISASB2 & SB & 0.74 & .09 & 8.02 & .001 & 1.74 \\
\hline ISASB3 & SB & 0.68 & .05 & 14.73 & .001 & 2.17 \\
\hline ISASB4 & SB & 0.95 & .07 & 13.155 & .001 & 2.24 \\
\hline ISASB5 & $\mathrm{SB}$ & 1.23 & .10 & 12.19 & .001 & 2.27 \\
\hline ISASB6 & SB & 1.15 & .07 & 16.39 & .001 & 2.47 \\
\hline ISASB7 & SB & 0.97 & .06 & 16.95 & .001 & 1.87 \\
\hline ISASB8 & SB & 0.77 & .06 & 12.84 & .001 & 2.31 \\
\hline ISASB9 & SB & 1.01 & .09 & 10.92 & .001 & 2.65 \\
\hline ISASB10 & SB & 0.83 & .06 & 14.17 & .001 & 1.67 \\
\hline ISASB11 & SB & 0.98 & .08 & 12.08 & .001 & 1.97 \\
\hline
\end{tabular}

revelam não haver multicolineariedade, uma condição observada no VIF (definida em português como "Inflação da Variância do Fator") com base no fator de tolerância entre as variáveis, o qual, deverá apresentar escores que não excedam um valor de 5 (Hair et al., 2009). Sendo assim, observou-se que o VIF variou de 1.20 a 3.73 ( $p<$ .01), revelando não existir multicolinearidade, o que contribui para a estimação do modelo pretendido.

Considerando tais achados, eles corroboram a estrutura fatorial composta por dois fatores: sexismo hostil e benévolo. Observou-se que as associações Lambdas $(\lambda)$ entre os 
fatores revelaram boa força associativa. Esses resultados se confirmam pela observação das estimativas de predição do modelo proposto, a partir da análise de regressão, as quais identificam as variáveis como significativas e com uma razão critério que esteve dentro do intervalo (Hair et al., 2009).

Tendo confirmado a estrutura fatorial comprovada nas referidas amostras, procurou-se verificar a relação entre sexismo ambivalente (hostil e benévolo) e a idade, o tempo de atuação e a identificação com a área de atuação profissional. A partir de uma correlação Pearson, os resultados foram os seguintes: a variável idade, ainda que tenha revelado escores fracos, apresentou uma correlação positiva e significativa com o sexismo ambivalente na pontuação total $(r=.13 ; p<.01)$, no sexismo hostil $(r=0.12 ; p<.01)$ e no benévolo $(r=$ $0,12 ; p<.01)$. Quanto ao tempo de formação, não houve correlação significativa.

Das 22 áreas de atuação listadas, o/a respondente indicava em uma escala de cinco pontos o quanto cada uma representava seu trabalho naquele momento. Isso permitiu que se analisassem as associações entre a identificação com as áreas e o sexismo ambivalente $(\mathrm{SA})$, sexismo hostil ( $\mathrm{SH})$ e sexismo benévolo $(\mathrm{SB})$. As correlações foram significativas $(p$ $<.05)$ e positivas com as seguintes áreas: Avaliação Psicológica $\left(r_{\mathrm{SA}}=0.21 ; r_{\mathrm{SH}}=0.19 ; r_{\mathrm{SB}}=\right.$ 0.19), Neuropsicologia e Neurociência do Comportamento $\left(r_{\mathrm{SA}}=0.24 ; \mathrm{rSH}=0.18 ; r_{\mathrm{SB}}=\right.$ 0.22), Psicologia do Esporte $\left(r_{\mathrm{SA}}=0.19 ; r_{\mathrm{SH}}=0.19 ; r_{\mathrm{SB}}=0.16\right)$, Psicologia do Trânsito $\left(r_{\mathrm{SA}}\right.$ $\left.=0.13 ; r_{\mathrm{SH}}=0.13 ; r_{\mathrm{SB}}=0.11\right)$, Psicologia Experimental $\left(r_{\mathrm{SA}}=0.21 ; r_{\mathrm{SH}}=0.17 ; r_{\mathrm{SB}}=0.21\right)$, Psicologia Organizacional e do Trabalho $\left(r_{\mathrm{SA}}=0.13 ; r_{\mathrm{SH}}=0.11 ; r_{\mathrm{SB}}=0.11\right)$. Por outro lado, uma única área apresentou correlações negativas. Para a Psicologia Social e Comunitária, os escores foram $r_{\mathrm{SA}}=-0.13$ e $r_{\mathrm{SH}}=-0.16$, não havendo correlação significativa para $\mathrm{O}$ sexismo benévolo.

\section{Discussão}

De forma geral, nesta pesquisa, pretendeu-se verificar a qualidade empírica da medida do sexismo ambivalente em uma amostra de psicólogos/as no Brasil, a partir da hipótese de consistência do seu modelo bifatorial. Acredita-se que, não apenas comprovou-se a estrutura psicométrica pretendida, mas os resultados observados, teoricamente, permitem refletir sobre a existência de sexismo na referida amostra. Isso sugere que, embora seja reprimido pelas diretrizes da profissão (Conselho Federal de Psicologia, 2005), essa forma de preconceito está presente no universo profissional da Psicologia, permitindo afirmar o quanto as conduções discriminatórias presentes na sociedade também estão inseridas na dinâmica da atuação desses/as profissionais. Essa reflexão se sustenta na avaliação dos indicadores estatísticos, os quais têm como base diferentes evidências empíricas (p.ex., $\chi^{2}$ ) $g l$, RMR, GFI, AGFI, NFI, CFI, RMSEA). Além disso, observou-se que o modelo fatorial proposto sobre o sexismo ambivalente se mostrou adequado, corroborando a hipótese pretendida, a qual não apenas sustenta a qualidade psicométrica da escala sobre sexismo, mas, também, revela o poder explicativo desse constructo em uma amostra tão específica como profissionais de Psicologia.

Apesar da garantia na relação item-fator, na análise exploratória e as correlações internas entre fatores de cada escala encontrada nos estudos anteriores (Glick e Fiske, 1996; Formiga et al., 2002; Ferreira, 2004; Formiga, 2005), os cálculos realizados por esses 
autores se baseiam apenas nos dados obtidos, desconsiderando um modelo teórico fixado, capaz de orientar a extração das dimensões latentes, justamente, porque nas análises exploratórias não é possível apresentar qualquer indicação sobre a bondade de ajuste do modelo. As técnicas de análise de modelagem estrutural têm a vantagem de levar em conta a teoria para definir os itens pertencentes a cada fator, bem como, apresenta indicadores de bondade de ajuste que permitem decidir objetivamente sobre a validade de constructo da medida analisada.

Em relação à avaliação de associações entre sexismo ambivalente e idade, tempo de atuação e identificação com áreas de atuação, os resultados chamam a atenção para o último. A única correlação negativa entre o sexismo e o quanto o/a profissional se identificava com cada área foi para Psicologia Social e Comunitária. A associação entre quanto um/a psicólogo/a se identifica com as áreas de atuação também foi avaliada em relação a outras medidas de preconceito. No estudo de Gaspodini e Falcke (2018a), o preconceito contra a diversidade sexual e de gênero, uma medida de preconceito extremo, também mostrou correlações negativas com a Psicologia Social e Comunitária, além de áreas afins, como Análise Institucional, Psicologia Política e Psicologia da Saúde. Tal resultado evidencia que a dedicação às demandas sociais está associada à menor discriminação e preconceito. Além disso, A Psicologia Social e Comunitária se dedica ao estudo dos processos de preconceito e discriminação, tendo sido pioneira nos estudos de gênero dentro do campo da Psicologia (Santos, Carvalho, Amaral, Borges, \& Mayorga, 2016). Apesar de tais resultados contribuírem para uma reflexão a respeito do tema e área científica, sugerese parcimônia nestas avaliações, já que, mesmo sendo significativas as correlações, elas tiveram escores baixos tamanhos de efeito e não indicam causalidade.

Quanto à idade e ao tempo de atuação, a idade apresentou relação positiva e significativa, o que representou um resultado não esperado, já que se reflete sobre o desenvolvimento do sujeito na formação psicológica em termos de melhores condições para poder gerir os conflitos interpessoais. Todavia, parece que o fator geracional evidencia maior impregnação de estereótipos em pessoas com mais idade, evidenciando uma possível desconstrução nas novas gerações. Tendo por base esses resultados, a principal contribuição ocorre quando se observa que os profissionais de Psicologia, em distintas áreas de formação, não estão para além do bem e do mal na manifestação dos fenômenos discriminatórios. Tal resultado rompe com a construção de uma estereotipia presente na sociedade quanto ao/à psicólogo/a ser 'aquele' indivídiduo livre de tudo e aberto a todos, sempre compreensível e totalmente centrado no equilíbrio das emoções pessoais.

De forma geral, destaca-se a garantia do modelo bifatorial oblíquo ajustado, constructo capaz de mensurar o sexismo e, principalmente, a sua forma direta (hostil) e indireta (sutil), a qual, provavelmente, de acordo com Formiga et al. (2002), legitimam os mecanismos de manutenção da desigualdade de gênero e que visa as relações de domínio por parte dos homens frente às mulheres, o que é reconhecido pelos próprios profissionais de Psicologia. O ISA, na distinta amostra, evidencia o preconceito frente às mulheres, ainda que a maior parte da amostra seja composta por elas (79.7\%), mas, é preciso salientar que todo esse percentual é composto por psicólogas. 
Com efeito, este estudo permitiu evidenciar que o sexismo hostil e benévolo além de estar presente em uma amostra de profissionais de Psicologia, foi capaz de avaliar que o preconceito frente às mulheres ainda se mantém frente à desigualdade de gênero, mesmo tendo sido a amostra composta majoritariamente por mulheres. Essa medida não tem somente o objetivo de avaliar a qualidade do instrumento de medida sobre o fenômeno do preconceito, mas, também, apontar a necessidade de uma reflexão por parte dos/as profissionais e discentes na área da ciência psicológica, de que esse é um fenômeno ainda existente, independente do contexto social, político e, neste espaço da pesquisa, o profissional.

Apesar de os resultados revelarem indicadores psicométricos consistentes e confiáveis, é preciso destacar algumas limitações do presente estudo. Uma delas se refere à necessidade de reunir evidências adicionais da validade, precisão e consistência interna da medida de sexismo, as quais deverão contemplar validade de critério ou convergente em relação a constructos correlatos (p.ex., sobre o preconceito em relação às mulheres, violência contra as mulheres, coerção sexual, etc.). Também seria útil conhecer a discriminação, representatividade e a estabilidade temporal (teste-reteste) da presente medida em amostras de profissionais formados recentemente na área (por exemplo, menos de 1 ano). Por fim, seria não menos importante, replicar o ISA em amostras mais diversificadas quanto à comparação do profissional com dedicação exclusiva na atuação psicológica e aquele que acumula profissões (atua como psicólogo e tem outra função profissional), entre outras possibilidades de investigação.

\section{Referências}

Bilich, F., da Silva, R., \& Ramos, P. (2006). Análise de flexibilidade em economia da informação: Modelagem de equações estruturais. Revista de Gestão da Tecnologia e Sistemas de Informação, 3(2), 93-122. Recuperado de http://www.scielo.br/pdf/jistm/v3n2/03.pdf

Byrne, B. M. (1989). A primer of LISREL: Basic applications and programming for confirmatory factor analytic models. New York, NY: Springer-Verlag.

Camino, L. (1998). Direitos humanos e Psicologia. In Comissão Nacional de Direitos Humanos do Conselho Federal de Psicologia (Ed.), Psicologia, ética e direitos humanos (pp. 39-63). Brasília, DF: Comissão Nacional de Direitos Humanos do Conselho Federal de Psicologia.

Campana, A. N. N. B., Tavares, M. C. G. C. F., Silva, D., \& Diogo, M. J. D. (2009). Translation and validation of the Body Image Avoidance Questionnaire (BIAQ) for the Portuguese language in Brazil. Behavior Research Methods, 41(1), 236-243. doi: 10.3758/BRM.41.1.236

Conselho Federal de Psicologia. (2005). Código de ética profissional do psicólogo. Brasilia, DF: Autor.

Conselho Federal de Psicologia. (2019). A psicologia brasileira apresentada em números. Recuperado de http://www2.cfp.org.br/infografico/quantos-somos

Faul, F., Erdfelder, E., Buchner, A., \& Lang, A. G. (2009). Statistical power analyses using G*Power 3.1: Tests for correlation and regression analyses. Behavior Research Methods, 41(4), 1149-1160. doi: 10.3758/brm.41.4.1149 
Ferreira, M. C. (2004). Sexismo hostil e benevolente: Inter-relações e diferenças de gênero. Temas em Psicologia, 12(2), 119-126. Recuperado de http://pepsic.bvsalud.org/ $\mathrm{pdf} / \mathrm{tp} / \mathrm{v} 12 \mathrm{n} 2 / \mathrm{v} 12 \mathrm{n} 2 \mathrm{a} 04 . \mathrm{pdf}$

Formiga, N. S. (2005). Estrutura fatorial do Inventário de Sexismo Ambivalente a partir da análise dos eixos principais. Psicólogo informação, 9(9), 9-28.

Formiga, N. S. (2007). Valores humanos e sexismo ambivalente. Revista do Departamento de Psicologia - UFF, 19(2), 381-396. doi:10.1590/s010480232007000200009

Formiga, N. S. (2011). Inventário de Sexismo Ambivalente: Um estudo a partir da modelagem de equação estrutural. Revista de Psicologia, 2(1), 104-116. Recuperado de http://200.129.29.202/index.php/psicologiaufc/article/view/79/78

Formiga, N. S. (2015). Evidência psicométrica de um modelo fatorial-conceitual concorrente do Inventário de Sexismo Ambivalente em brasileiros. Revista de Psicología, 17(1), 9-20. doi: 10.18050/revpsi.v17n1a1.2015

Formiga, N. S. \& Camino, L. (2001). A dimensão do inventário de papéis sexuais (BSRI): A masculinidade e feminilidade em universitários. Estudos de Psicologia, 18(2), $41-$ 49. doi: 10.1590/S0103-166X2001000200004

Formiga, N. S., \& da Silva Neta, A. B. (2009). Precisão preditiva das novas formas de sexismo a partir das orientações valorativas em brasileiros. Psico, 40(2), 174183. Recuperado de http://revistaseletronicas.pucrs.br/fo/ojs/index.php/ revistapsico/article/view/4038

Formiga, N. S., Gouveia, V. V., \& Santos, M. N. (2002). Inventário de Sexismo Ambivalente: Sua adaptação e relação com o gênero. Revista Psicologia em Estudo, 7(1), 105-111. doi: 10.1590/s1413-73722002000100013

Formiga, N. S., Guedes, J. R., Silva, R. G. da, \& de Oliveira, M. R. F. (2012). A mensuração do sexismo ambivalente: Manutenção da sua evidência psicométrica em jovens. Recuperado de http://www.psicologia.pt/artigos/textos/A0658.pdf

Formiga, N. S., Omar, A. G., \& Vaamonde, J. D. (2009). Atitudes frente aos grupos minoritários em brasileiros: Análise fatorial confirmatória e modelagem de equação estrutural. Encontro - Revista de Psicologia, 12(17), 71-84. Recuperado de http://www.pgsskroton.com.br/seer/index.php/renc/article/view/2547

Gaspodini, I. B., \& Falcke, D. (2018a). Relações entre preconceito e crenças sobre diversidade sexual e de gênero em psicólogos/as brasileiros/as. Psicologia: Ciência e Profissão, 38(4), 744-757. doi: 10.1590/1982-3703001752017

Gaspodini, I. B., \& Falcke, D. (2018b). Sexual and gender diversity in clinical practice in psychology. Paidéía, 28, e2827. doi: 10.1590/1982-4327e2827

Giovannetti, A. (2009). 60 anos da declaração universal dos direitos humanos: Conquistas do Brasil. Brasília, DF: Fundação Alexandre Gusmão. 
Glick, P., \& Fiske, S. T. (1996). The Ambivalent Sexism Inventory: Differentiating hostile and benevolent sexism. Journal of Personality and Social Psychology, 70(3), 491-512. doi: 10.1037/0022-3514.70.3.491

Glick, P., \& Fiske, S. T. (2001). An ambivalent alliance: Hostile and benevolent sexism as complementary justifications for gender inequality. American Psychologist, 56(2), 109-118. doi: 10.1037/0003-066x.56.2.109

Hair, J. F., Black, W. C., Babin, B. J., Anderson, R. E., \& Tatham, R. L. (2009). Análise multivariada de dados (6a ed.). Porto Alegre, RS: Bookman.

Joreskög, K., \& Sörbom, D. (1989). LISREL 7 user's reference guide. Mooresville, NC: Scientific Software.

Mladinic, A., Saiz, J. L., Díaz, M., Ortega, A., \& Oyarce, P. (1998). Sexismo ambivalente en estudiantes universitarios chilenos: Teoría, medición y diferencias de género. Revista de Psicología Social y Personalidad, 14, 1-14.

Myers, D. G. (2014). Psicologia social (10a ed.). São Paulo, SP: McGraw Hill Education.

Organização das Nações Unidas. (1948). Declaração universal dos direitos humanos. Genebra, Suíça: Autor.

Pereira, A., Monteiro, M. B., \& Camino, L. (2009). Social norms and prejudice against homosexuals. Spanish Journal of Psychology, 12(2), 576-84. doi: 10.1017/ s1138741600001943

Pescosolido, B. A., \& Martin, J. K. (2015). The stigma complex. Annual Review of Sociology, 41(1), 87-116. doi: 10.1146/annurev-soc-071312-145702

Pettigrew, T. F., \& Meertens, R. W. (1995). Subtle and blatant prejudice in Western Europe. European Journal of Social Psychology, 25(1), 57-75. doi: 10.1002/ejsp.2420250106

Pettigrew, T. F., \& Meertens, R. W. (2001). In defense of the subtle prejudice concept: A retort. European Journal of Social Psychology, 31(3), 299-309. doi: 10.1002/ejsp.45

Santos, L. C. dos, Carvalho, A. B., Amaral, J. G., Borges, L. A., \& Mayorga, C. (2016). Gênero, feminismo e psicologia social no Brasil: Análise da revista Psicologia \& Sociedade (1996-2010). Psicologia \& Sociedade, 28(3), 589-603. doi: 10.1590/1807-03102016v28n3p589

Valentini, F., \& Damásio, B. F. (2016). Variância média extraída e confiabilidade composta: Indicadores de precisão. Psicologia: Teoria e Pesquisa, 32(2), 1-7. doi: 10.1590/0102$3772 \mathrm{e} 322225$

Vijver, F. J. R. van de, \& Leung, K. (1997). Methods and data analysis for cross-cultural research. Thousand Oaks, CA: Sage Publications. 